\title{
Alterstice
}

Revue internationale de la recherche interculturelle

International Journal of Intercultural Research

Revista International de la Investigacion Intercultural

\section{Récits de souffrance et VIH/sida : réflexions sur quelques enjeux anthropologiques et éthiques du témoignage}

\section{Bertrand Lebouché et Joseph Josy Lévy}

Volume 1, numéro 2, 2011

Droits humains et témoignages : l'épreuve de la culture

URI : https://id.erudit.org/iderudit/1077606ar

DOI : https://doi.org/10.7202/1077606ar

Aller au sommaire du numéro

Éditeur(s)

Alterstice

ISSN

1923-919X (numérique)

Découvrir la revue

Citer cet article

Lebouché, B. \& Lévy, J. J. (2011). Récits de souffrance et VIH/sida : réflexions sur quelques enjeux anthropologiques et éthiques du témoignage. Alterstice, 1(2), 97-107. https://doi.org/10.7202/1077606ar
Résumé de l'article

Comme le montrent les travaux sur la maladie grave, celle-ci constitue un événement majeur qui affecte non seulement l'organisme mais aussi la perception de l'identité, et conduit les individus à devenir étrangers ou invisibles, rendant difficile l'expression du témoignage de l'expérience de leur maladie. Ces caractéristiques sont pleinement illustrées chez les personnes vivant avec le VIH/sida, jusqu'à disparaître souvent des espaces de dépistage, de diagnostic et de soins mis à leur disposition. Ces enjeux son repris à partir de l'approche éthique de Johannes-Baptist Metz et de William T. Cavanaugh qui offrent un cadre de réflexion qui met au centre de leur argumentation les motifs éthiques touchant les espaces de réappropriation des récits de souffrance et leur réinscription dans l'espace social, en particulier dans le contexte interculturel. À partir de ces approches, les espaces de soins font l'objet d'une nouvelle interprétation, montrant comment ils peuvent devenir des lieux de réflexion, d'échanges et de pratiques qui dépassent le simple accès à des traitements biomédicaux, comme les antirétroviraux. Ces innovations, paradoxalement, font rebondir le statut du récit et du témoignage pour les personnes vivant avec le $\mathrm{VIH} /$ sida. 


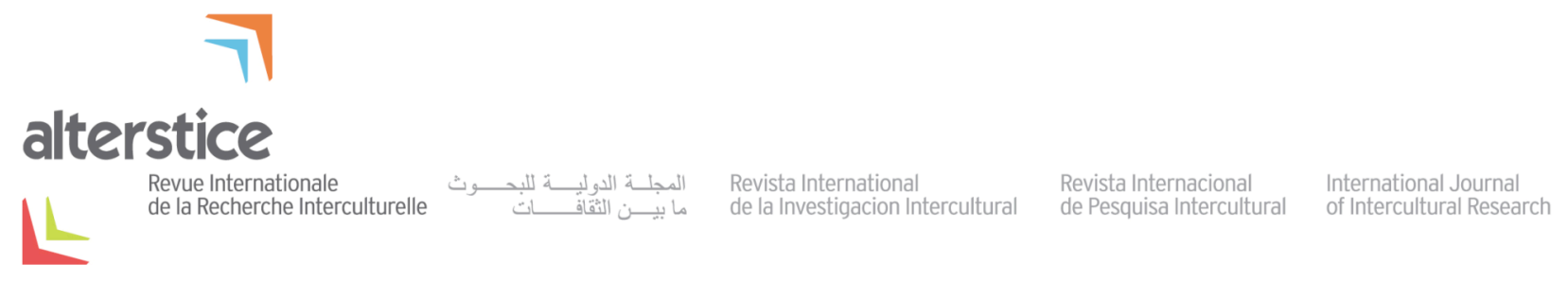

ARTICLE THÉMATIQUE

\section{Récits de souffrance et $\mathrm{VIH} /$ sida : réflexions sur quelques enjeux anthropologiques et éthiques du témoignage}

Bertrand Lebouché ${ }^{1}$ et Joseph Josy Lévy ${ }^{2}$

\section{Résumé}

Comme le montrent les travaux sur la maladie grave, celle-ci constitue un événement majeur qui affecte non seulement l'organisme mais aussi la perception de l'identité, et conduit les individus à devenir étrangers ou invisibles, rendant difficile l'expression du témoignage de l'expérience de leur maladie. Ces caractéristiques sont pleinement illustrées chez les personnes vivant avec le VIH/sida, jusqu'à disparaître souvent des espaces de dépistage, de diagnostic et de soins mis à leur disposition. Ces enjeux son repris à partir de l'approche éthique de Johannes-Baptist Metz et de William T. Cavanaugh qui offrent un cadre de réflexion qui met au centre de leur argumentation les motifs éthiques touchant les espaces de réappropriation des récits de souffrance et leur réinscription dans l'espace social, en particulier dans le contexte interculturel. À partir de ces approches, les espaces de soins font l'objet d'une nouvelle interprétation, montrant comment ils peuvent devenir des lieux de réflexion, d'échanges et de pratiques qui dépassent le simple accès à des traitements biomédicaux, comme les antirétroviraux. Ces innovations, paradoxalement, font rebondir le statut du récit et du témoignage pour les personnes vivant avec le $\mathrm{VIH} /$ sida.

\section{Rattachement des auteurs}

${ }^{1}$ CTN/CIHR, Centre universitaire de santé McGill, Montréal, Canada

2 Département de sexologie, Université du Québec à Montréal, Montréal, Canada

\section{Correspondance}

bertrand.lebouche@gmail.com; josyjosephlevy@ymail.com

\section{Mots clés}

infection par le VIH/sida; éthique; expérience de la maladie; disparition; témoignage; étrangeté

\section{Pour citer cet article :}

Lebouché, B. et Lévy, J. (2011). Récits de souffrance et VIH/sida : réflexions sur quelques enjeux anthropologiques et éthiques du témoignage. Alterstice, 1(2), 97-108. 


\section{Introduction}

La maladie, surtout quand elle est grave, interpelle de façon significative à la fois les sociétés et les personnes atteintes. Elle constitue ainsi une forme élémentaire d'événement (Augé, 1984; Bastide, 1996), qui s'accompagne d'interprétations et de significations multiples (Laplantine, 1993). Bensa et Fassin (2002) notent dans cette perspective que l'événement ne se présente pas dans sa vérité objective mais qu'il est construit, se manifeste et se met en scène en particulier dans le témoignage ${ }^{1}$ de celles et ceux qui ont traversé cet événement. C'est ainsi le cas des épidémies qui constituent des moments privilégiés révélateurs des valeurs et des contradictions propres à une société. Cette perspective se retrouve dans le cas du VIH/sida qui, à cause de ses rapports à la maladie, à la sexualité et à la mort, touche les préoccupations fondamentales d'une société, provoquant ainsi une " épidémie de significations " (Ménil, 1997). Même si aujourd'hui la maladie semble maîtrisée avec l'apparition de traitements antirétroviraux de plus en plus efficaces, la peur de la transmission continue d'alimenter des formes de stigmatisation et de discrimination, en particulier à l'encontre de minorités sexuelles ou ethnoculturelles, contribuant à renforcer leur étrangeté et leur marginalisation. Dans ces conditions, la vie sociale, affective et relationnelle des personnes vivant avec le $\mathrm{VIH} /$ sida est profondément affectée et elles se retrouvent souvent incapables de se faire entendre dans un espace public réfractaire au récit de leur expérience et à leur témoignage, ce qui entraîne leur invisibilité.

Dans cet article, nous explorerons, en premier lieu, certaines des conditions nécessaires à l'expression du témoignage et du récit dans un cadre général. En second lieu, nous verrons les conditions de l'expression de ces formes narratives dans le cadre de l'infection par le VIH/sida.

\section{Le récit et le témoignage et leurs conditions d'expression}

Le récit, comme l'ont montré de nombreux travaux (Laplantine, Lévy, Martin et Nouss, 1998), constitue un élément essentiel de l'expression humaine. II participe à la construction du sujet dans la mesure où celui qui raconte devient acteur de la parole (Metz, 1979, p. 240). Le récit est ainsi "la forme par laquelle l'humain se saisit de I'hétérogénéité de son expérience et lui attribue sens et cohérence " (Parent, 2006, p. 113). Si le récit se fonde sur une expérience personnelle, le témoignage dépasse la narration du vécu pour s'ouvrir à la compréhension d'un événement plus large dont le narrateur devient le garant. Le témoignage s'arrime donc à la recherche d'une vérité à inscrire dans la mémoire collective par une prise de parole publique. Cependant, ces deux formes narratives ne peuvent faire l'économie de certains éléments fictionnels, ce qui rend difficile un rapport objectif aux faits et il subsiste aussi une part d'indicible qui signale l'impossibilité des mots à rendre le tout de l'expérience vécue. Comme le souligne Carasso (2001), en parlant du témoignage dans l'œuvre de Primo Levi, son statut reste problématique dans la mesure où le témoin n'est pas dans un rapport objectif face à l'événement et la réalité qu'il observe mais il en est partie inclusive et incontournable. Mais l'expression de ces formes narratives peut être bloquée par certaines conditions sociales et psychologiques qui empêchent alors leur énonciation, leur écoute et leur partage.

En premier lieu, on peut noter les difficultés qui sont rattachées au contexte socioculturel où les modes de narrativité et d'expressions des éléments autobiographiques et l'intensité des affects (honte, humiliation et culpabilité par exemple) sont susceptibles d'intervenir sur le contenu du récit en l'amputant de références à des situations ou de références considérées comme taboues et comme transgressant des interdits sociaux. Les normes langagières et leurs connotations implicites et explicites, de même que les formes narratives privilégiées peuvent contraindre la structure du récit. Ces difficultés peuvent être particulièrement exacerbées dans un contexte interculturel, où la communication des récits se heurte souvent à des incompréhensions liées au manque de connaissances réciproques des codes culturels qui régissent les interactions ainsi qu'à des problèmes linguistiques et de traductions. Ces problèmes se retrouvent par exemple dans le contexte de la communication médecinpatient, chacun appartenant à un univers culturel différent et pouvant de surcroît être sensible à des dimensions différentes de la maladie : le médecin fait attention aux dimensions symptomatiques liées au disease tandis que le patient rend compte de son expérience de la maladie comme illness (Kleinmann, 1972; Maman, 2009).

\footnotetext{
${ }^{1}$ Nous verrons plus avant, avec Metz, l'importance du témoignage et la nécessité de laisser émerger les histoires de souffrances.
} 
En second lieu, le récit et le témoignage exigent, lorsqu'ils font référence à des expériences extrêmes, des espaces où la prise de parole peut se faire dans des conditions d'écoute et d'acceptation, ce qui nécessite l'établissement de liens de confiance et de sécurité. En effet, le sujet porteur du récit se voit amener à renoncer à son invisibilité et à son anonymat, pour prendre la parole en public, en assumant ainsi le risque du dévoilement et donc du regard de l'autre ${ }^{2}$ sur son statut. II accepte dans ces conditions de poser un geste sociopolitique plus engagé. Arendt, en particulier développe cette perspective à partir des concepts d'apparence et d'apparition en les arrimant à la notion d'espace public. Selon elle, les « hommes apparaissent en paroles et en acte et indiquent par là comment ils entendent paraître, et ce qui, à leur avis, est digne ou non d'être vu. » (Arendt, 2005, p. 56, cité par Noali, 2009, p. 2). Cette apparition nécessite un espace démocratique - espace public -, où l'on peut rencontrer les autres humains dans une certaine liberté (Arendt, 1972) et trouver sa place par la parole ou par ses actes comme citoyen. Cet espace est la scène indispensable à la " production théâtrale " où chacun peut librement se mettre en scène tel qu'il l'entend. II n'est pas sûr que ces conditions nécessaires à l'expression du récit et du témoignage soient présentes dans le cadre du VIH/sida.

\section{Un difficile témoignage au temps du VIH/sida}

Sur le plan socioculturel, l'irruption du $\mathrm{VIH} /$ sida en tant que manifestation de l'imprévu et de l'inattendu dans le tissu social n'est pas sans répercussions sur les représentations sociales de la maladie et sur les conduites face aux personnes vivant avec elle. Ainsi, lors de son apparition et même après la découverte du virus responsable, les perspectives biomédicales sur l'origine du VIH/sida ont été débordées par un ensemble de représentations complexes quant aux mécanismes de contamination et liées à des motifs sexuels, religieux et moraux. La maladie a par exemple été associée au style de vie homosexuel, d'où l'étiquette de "peste gaie " qui lui a été accolée aux débuts de l'épidémie (Lévy et Nouss, 1994). Si ce référent s'est effacé, les notions archaïques de souillure, associées aux concepts de mal, de malédiction et de maléfice et au mécanisme de bouc-émissaire (stigmatisation des "groupes à risques », homosexuels, drogués, Haïtiens et hémophiles) ont aussi nourri les représentations sociales.

Ces représentations se sont cependant estompées à partir de 1996 avec l'apparition des traitements antirétroviraux qui ont contribué à resituer le $\mathrm{VIH} / \mathrm{sida}$ dans le cadre des maladies chroniques et à atténuer son caractère exceptionnel, émoussant ainsi certaines des conduites sociales de discrimination et de stigmatisation à l'encontre des personnes vivant avec le $\mathrm{VIH} /$ sida, sans toutefois les faire disparaître de tous les milieux sociaux. Pour ces dernières, cependant, le diagnostic d'une contamination au VIH/sida constitue un évènement marquant qui les oblige à revoir la signification qu'elles donnent à leur existence, à « rechercher une nouvelle familiarité avec son propre corps qui ne cesse de devenir étranger » (Langlois, 2006, p. 276) et à réorganiser les modalités de leurs liens sociaux et familiaux (Denzin, 1989; Lévy et collab., 2002; Lévy, Pierret, 2006; Pierret et Trottier, 2004).

Cette question de l'étrangeté et de l'exil de soi est ainsi au centre de l'expérience de la maladie. Pour beaucoup, l'expérience de la maladie grave, perçue comme exil, se traduit par des pertes. La dépossession de la santé pour la personne devenue malade s'accompagne d'une extirpation de soi, géographique et temporelle, qui touche les sphères physiques et mentales (David, 2004; Porée, 1993) et qui la rend étrangère aux yeux de son entourage. Cette « inquiétante étrangeté " peut être exacerbée par les interventions cliniques et de soins (Louis-Courvoisier, 2005, Nancy, 2000). Par ailleurs, la contamination peut devenir source de honte et, lorsque l'entourage exprime des attitudes stigmatisantes face à des activités sexuelles jugées inacceptables ou face à la maladie et aux malades, la révélation de la séropositivité dans l'espace social devient alors difficile, ce qui pousse à cacher la maladie, souvent asymptomatique par ailleurs, d'où la nécessité d'une gestion du secret (Langlois, 2006) et du dévoilement qui obéit à des stratégies diverses selon les personnes et les réseaux sociaux impliqués.

Même si les mouvements de lutte contre le $\mathrm{VIH} /$ sida ont pu donner une image de forte solidarité et de cohésion, l'expérience du $\mathrm{VIH} /$ sida comme paradigme de la maladie sécularisée (Langlois, 2006) peut renvoyer les personnes vivant avec le $\mathrm{VIH} /$ sida à leur solitude, sans chemin tracé d'avance, avec l'obligation d'apprendre par elles-mêmes

\footnotetext{
${ }^{2}$ Comme l'indique le site de la COCQ-SIDA, "le témoignage est un puissant outil de sensibilisation pouvant faire, socialement, une différence. Dans le domaine du VIH/sida, témoigner, c'est nourrir le combat contre la stigmatisation et la discrimination. » [En ligne]. http://www.cocqsida.com/simpliquer/temoigner.html (page consultée le $1^{\text {er }}$ juin 2011).
} 
le métier de malade, exposées au regard des autres, condamnées à vivre parmi les personnes non atteintes et devenant pour les autres une énigme, voire une menace (Langlois, 2006). C'est pourquoi nombre de personnes vivant avec le $\mathrm{VIH} /$ sida choisissent de taire leur maladie afin d'éviter les discriminations, se retrouvant en conséquence dans une situation d'exclusion et d'invisibilité. De plus, la multiplicité des vulnérabilités qu'elles traversent témoigne que, dans les sociétés contemporaines, l'inscription de ces sujets dans l'humanité est loin d'être assurée, d'où leur précarité, dont l'une des conséquences est la disparition. Cette fragilité anthropologique de l'inscription dans l'humain remet en cause l'évidence du lien social et rend compte du caractère négatif qui le hante (Le Blanc, 2006) dans la mesure où la personne est privée d'une reconnaissance fondamentalement nécessaire à son existence, reconnaissance qui, par son absence, crée de vives souffrances liées au mépris auquel elle est confrontée ou qu'elle appréhende (Caillé, 2007).

Ainsi, depuis près de trente ans, les maladies liées au VIH/sida se construisent " sous nos yeux " dans l'espace public, et pourtant certaines personnes vivant avec échappent au regard, deviennent invisibles, comme c'est le cas pour les femmes séropositives (Mensah, 2003). L'absence ou le manque de lieux d'expression des formes contemporaines de la sexualité et le manque d'accès à des stratégies de préventions adaptées incitent certains à adopter des conduites à risque pour le VIH/sida. Cette invisibilité s'accompagne de l'absence d'expression du récit ou de témoignage. On peut prendre ici comme exemple le milieu afro-américain ou africain où les contextes culturels et sociosexuels laissent très peu de place à l'acceptation des minorités sexuelles et à leur expression. Les personnes d'orientation homosexuelle ou bisexuelle doivent ainsi cacher leur orientation et apparaître aux autres conformes aux attentes sociales ${ }^{3}, c^{\prime}$ est-à-dire hétérosexuelles et mariées, et projeter cette image tout en menant une double vie dont une partie n'est souvent jamais exprimée ou partagée. Ces hommes ont ainsi recours à des pratiques sexuelles clandestines avec d'autres hommes, souvent non protégées, dans des contextes fortement à risque, et ces espaces parallèles sont d'autant plus importants qu'ils n'ont pas la maîtrise de leur intimité, une situation que l'on retrouve dans le contexte carcéral où les espaces de disparition sont toujours plus difficiles à contenir et à maîtriser que les espaces d'apparition (Noali, 2009).

Les stratégies de contournement sont alors dangereuses pour l'entourage, en particulier pour la conjointe mariée, et constituent un casse-tête pour les instances de la santé publique qui ne peuvent assurer la validité des données épidémiologiques et l'adéquation des campagnes et des interventions de prévention. On peut citer ici l'exemple de

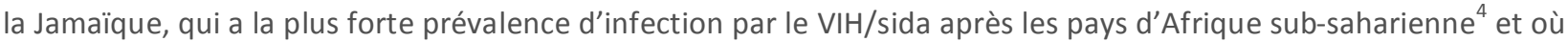
l'on retrouve une quasi " discrimination légalisée ${ }^{5}$ " de l'homosexualité, dans une société qui est foncièrement " anti-gay », ce qui incite ses membres à se marier rapidement et à avoir des enfants afin de légaliser au plus vite leur situation et s'affirmer publiquement comme non-gais ${ }^{6}$ (Fink, 2009). Les pratiques homosexuelles cachées avec des individus à haut risque peuvent disséminer ainsi rapidement le VIH/sida à la population générale. De plus, l'environnement social, politique ou religieux empêchant toute intervention de réduction des risques, les hommes qui ont des pratiques homosexuelles (HARSAH) n'ont accès ni aux moyens de prévention - comme les condoms -, ni aux services médicaux ou de prévention, qui pourraient les dénoncer. De ce fait n'existe aucune possibilité de

\footnotetext{
${ }^{3}$ On parle alors d'Afro-Américains on the down low, c'est-à-dire hétérosexuels avec leur partenaire principale, mais qui ont secrètement des partenaires HARSAH, c'est-à-dire des hommes qui ont des relations sexuelles avec d'autres hommes. Bond et collab. (2009) montrent que, contrairement à une opinion fortement répandue, ils ne seraient pas cependant les premiers agents de transmission de l'épidémie de $\mathrm{VIH} /$ sida. De fait, ils présentent moins de rapports anaux non protégés et sont moins fréquemment porteurs du VIH que les non down low.

${ }^{4} 32 \%$ des hommes qui ont des relations sexuelles avec d'autres hommes y sont porteurs du VIH.

5 " The reality in Jamaica is that "men who have sex with men, for fear of being prosecuted and being found guilty under the sodomy law, pretend that they're not gay," says Miriam Maluwa, the UNAIDS country representative for Jamaica, explaining how what she calls "legalized discrimination" has driven the HIV epidemic underground. "[Gay men] marry fairly rapidly, they have children fairly rapidly to regularize themselves, and that is really a ticking bomb." So we are really talking about this targeted group, having quite high levels of infections, which is interacting sexually with the general population. » (Fink, 2009).

${ }^{6}$ Fink (2009) explique que, pour désigner les gais, les Jamaïcains utilisent le mot fish et le chiffre number two - pour déféquer par rapport à number one pour uriner. Le refus de l'homosexualité est tel que ces deux termes ne sont plus utilisés par les jeunes, qui préfèrent le mot sea-creature ou qui refusent de prononcer le chiffre deux - pour number two-, ce qui pose des problèmes dans les écoles. De même, le terme battyman, utilisé pour désigner un gai, est la pire insulte que l'on puisse faire à un homme jamaïcain.
}

Alterstice-Revue Internationale de la Recherche Interculturelle, vol. 1, $n^{\circ} 2$ 
faire récit de sa vulnérabilité et de ses préoccupations. Dans ce cadre, face à l'impossibilité de vivre ouvertement son orientation, les pratiques clandestines ${ }^{7}$ peuvent être considérées comme autant de tentatives non seulement de préservation, mais aussi d'affirmation de soi. Même dans les contextes où les personnes vivant avec le VIH/sida ont accès à des soins de santé et à des traitements antirétroviraux satisfaisants, les intervenants en santé ne sont pas forcément ouverts à entendre le vécu de leurs patients, à cause des tabous entourant la sexualité.

De plus, avec la dominance des tests biomédicaux objectifs, les dimensions liées à l'expérience de la maladie et à sa construction personnelle ne constituent plus une priorité. Dans tous ces contextes, les personnes vivant avec le $\mathrm{VIH} /$ sida ne peuvent donc pas souvent partager leur expérience, rendant ainsi difficile l'expression des récits personnels, de souffrance ou de résilience, empêchant le témoignage ou l'amputant de ces dimensions sociosexuelles. Nous tenterons maintenant de cerner plus précisément les enjeux éthiques qui rendent nécessaire cette réappropriation du témoignage par les personnes vivant avec le $\mathrm{VIH} / \mathrm{sida}$.

\section{Quelle éthique pour la réapparition du témoignage de ces étrangers?}

L'approche éthique de Johannes-Baptist Metz et de William T. Cavanaugh nous offre un éclairage particulier sur les enjeux d'étrangeté, d'invisibilité et de disparition que traversent ces personnes vivant avec le VIH/sida et sur les conditions de repossibilisation de leur témoignage dans l’espace social.

\section{Une éthique fondamentale pratique}

La théologie politique de Johannes-Baptist Metz $^{8}$ offre une éthique ancrée dans le réel où l'histoire de souffrance des opprimés et des vaincus est source d'indignation et point de départ nécessaire à une réflexion éthique. La mise en récit des histoires de souffrance des disparus et des étrangers s'avère alors une condition essentielle pour fonder l'éthique car ces narrations, leur mise en mémoire et leur diffusion à travers des porte-voix des sans voix, même si ces témoignages sont une mémoire "dangereuse » pour les sociétés, ouvrent à la solidarité comme à la sensibilité à la souffrance des autres (Metz, 1979), qu'ils soient encore vivants ou qu'ils soient morts. Alors que le récit historique, habituellement, ne fait mémoire que des vainqueurs et fait disparaître les perdants, faire advenir I'histoire des vaincus permet de leur donner une place et empêcher qu'ils ne tombent dans l'oubli ou l'absence. Les récits des disparus restent ainsi premiers et deviennent condition de leur reprise par l'éthicien, qui peut alors fonder son discours argumentatif sur les éléments objectifs et subjectifs qu'ils révèlent (Jobin, 2006).

Cette éthique fondamentale pratique peut alors aider l'éthicien à prendre une place significative dans l'espace public, faire entendre des expériences significatives occultées et mettre en relief les carences sociales, politiques économiques et culturelles que les instances dominantes peuvent s'entêter à ne pas considérer ou à négliger. En faisant réapparaître les histoires de souffrance des humains, il contribue à une " anti-histoire " et à une analyse critique des stratégies qui escamotent la présence des plus faibles. L'approche de Metz nous semble essentielle dans le champ de l'intervention touchant le VIH/sida pour plusieurs raisons. Les intervenants en santé deviennent alors chargés, à l'instar des éthiciens, d'une responsabilité particulièrement importante, celle d'aider à la mise en récit de leurs patients et de devenir leur avocat pour rendre compte à la société, qui les mandate, des enjeux touchant les vulnérabilités spécifiques qui affectent ces patients. En ce sens, leur rôle social critique se voit élargi puisqu'ils auront à situer ces récits dans une analyse des conditions socioéconomiques, plus généralement des inégalités sociales de santé et des actions efficaces en santé publique, qui favorisent la diffusion du VIH/sida, rappelant par là que la santé des populations dépend davantage de l'amélioration de ces conditions que des avancées biomédicales (Callahan et Jennings, 2002).

\footnotetext{
${ }^{7}$ Noali les définit comme "un ensemble de conduites, voire de ficelles plus ou moins consciemment recherchées par le prisonnier en tant que moyens d'accéder à des espaces de liberté et d'intimité ou à leur illusion parce qu'elles permettent d'être soi-même, en soi et pour soi " (Noali, 2009, p. 12).

${ }^{8}$ Johannes Baptist Metz, théologien allemand contemporain, a développé une théologie politique qui a son origine dans son indignation face aux souffrances de nombre de ses contemporains, qui souvent sont considérés comme négligeables et qui sombrent dans l'oubli. Il a été fortement marqué comme allemand par l'histoire du nazisme et des camps comme Auschwitz.
} 
La bioéthique se doit donc de quitter le laboratoire et la relation singulière médecin-malade pour s'intéresser à des populations entières qui souffrent, dans des contextes marqués par des inégalités socioéconomiques et politiques. Le défi à relever ne concerne pas seulement la santé organique ou mentale des personnes vivant avec le VIH/sida, mais également le contexte structurel, qui rend des individus particulièrement vulnérables au $\mathrm{VIH} / \mathrm{sida}$ et qui augmente le risque de transmission et les empêche d'accéder aux moyens de prévention, de dépistage, de diagnostic ou de traitement. Une éthique de la santé au temps du VIH/sida ne peut donc pas faire l'économie d'une réflexion sur les structures sociales, économiques et politiques qui favorisent le risque d'une infection par le $\mathrm{VIH} / \mathrm{sida}$ (Keenan, 2005, p. 417). Les récits de vie et les témoignages occupent alors une fonction importante, celle d'éclairer des données épidémiologiques ou sociologiques en mettant l'accent sur le vécu et sur les itinéraires de maladie et de soins, permettant ainsi de rendre compte de l'expérience et de sa diversité.

Par exemple, du point de vue ethnoculturel, on retrouve une forte population originaire d'Afrique sub-saharienne et des Caraïbes dans les hôpitaux d'Europe et d'Amérique du Nord. La forte proportion de ces patients d'origine culturelle différente conduit nombre d'hôpitaux à chercher comment soigner autrement, et à surmonter ainsi l'étrangeté secondaire à la barrière linguistique ou à l'incompréhension devant une expérience de la maladie qui ne s'intègre pas dans une approche biomédicale et occidentale des soins. Dans les services spécialisés dans le suivi de I'infection par le $\mathrm{VIH} / \mathrm{sida}$, des malades provenant de différents groupes ethnoculturels (africain, asiatique, etc.) se côtoient et, surtout, ces services ont à accueillir des personnes vivant dans la plus grande précarité, souvent sans travail, parfois sans domicile. Miangu (2008) rapporte une expérience réussie de femmes médiatrices interculturelles de santé à l'hôpital d'Avicenne à Paris, où 70 pour cent de la population fréquentant le service de maladies infectieuses est étrangère. Il s'agit donc bien de trouver des médiateurs de santé capables de faire des liens entre ces mondes culturels et sociaux si différents. Ces médiateurs peuvent éviter au quotidien les conflits avec le personnel soignant et aider à faire accepter les soins et les contraintes liées au régime médicamenteux. Plus largement, ces médiateurs rendent possibles les témoignages des individus, afin que leur expérience ne compte pas pour rien et puisse contribuer à l'humanisation nécessaire des lieux de soins, et plus largement celle de la société. Ces médiateurs ont le rôle important de rendre compte du témoignage et d'en assurer la traduction et la transmission au personnel hospitalier. II n'est pas de sortie possible de l'étrangeté ou de l'invisibilité ni de mieuxêtre sans une prise en charge globale qui inclue l'appréciation du récit porteur du vécu.

\section{La disparition des corps et le passage d'un espace à l'autre}

Le théologien William T. Cavanaugh ${ }^{9}$, quant à lui, analyse les processus de disparition des corps dans le cas de l'usage de la torture ou du mapping qui enferme les sujets dans des lieux où leur parole est confisquée, ce qui entraîne la disparition des corps et des individus. Reprenant certaines des analyses de Certeau ${ }^{10}$, il caractérise les espaces comme devant être biographiques, c'est-à-dire modelés et réinvestis par l'histoire de chacun, dans la mesure où c'est leur récit même qui les crée. Selon Certeau (1990), quand le récit disparaît, il y a perte de l'espace et sa transformation en totalité informe, indistincte, en un lieu analogue à un tombeau, sans sortie possible. Le récit a alors un double impact sur l'espace : à l'intérieur et sur ses frontières. À l'intérieur, il permet un espace de parole et, aux frontières, il s'ouvre sur des points de rencontre mettant en évidence ce que Certeau (1990) qualifie de " paradoxe de la frontière : créés par des contacts, les points de différentiation entre deux corps sont aussi des points communs » (p. 186).

La frontière devient alors un symbole narratif d'échanges et de rencontres. Dans cette perspective, il ne s'agit donc pas de créer des lieux pour enfermer les étrangers dans nos sociétés, ni de les séparer de la société en fonction de leur dangerosité, mais de leur créer des espaces dont les frontières sont autant de points de rencontre. Comme l'explique Certeau (1990), alors que la carte découpe, le récit traverse. II transgresse même la frontière, comme une ouverture à l'altérité de l'étranger. Cette approche accorde une grande importance au fait que le récit puisse

\footnotetext{
9 Né en 1962, Cavanaugh est un théologien catholique américain brillant appartenant au courant de la nouvelle théologie politique en émergence dans le monde universitaire anglo-saxon. Il est disciple de Stanley Hauerwas, d'origine texane et méthodiste et l'un des théologiens américains actuellement les plus célèbres.

${ }^{10}$ Anthropologue, historien, philosophe et jésuite, Michel de Certeau (1925-1986) examine les pratiques spatialisantes, qui créent ce qu'il appelle des espaces. Dans son ouvrage L'invention du quotidien : L'art de plaire (Certeau, 1990), il élabore sur la manière dont les individus inventent leur quotidien, en détournant les objets et les codes à leur disposition.
} 
être dit et puisse ouvrir des points de passage avec le reste du corps social. Ces espaces biographiques deviennent habités par ceux qui étaient sans lieux. Leurs frontières entrent en relation avec les autres espaces biographiques, créant ainsi un véritable espace social de réapparition, comme un espace-temps du "bien-vivre ensemble " (Ladrière, 1996). On peut parler alors d'une configuration de l'espace social où les frontières entre les membres tombent, où tous, même ceux à la périphérie et à la marge, se retrouvent au centre : ils sont invités à témoigner, à créer un espace où les souffrances de chacun sont partagées par tous, et où ils ne se trouvent plus excommuniés de la société.

Cet espace de narrativité fait réapparaître les singularités des vaincus de notre société, et il leur permet de se reconstruire socialement et psychologiquement. Dans le domaine du VIH/sida, le mapping comporte cependant un danger, celui d'une lecture de santé publique trop "déterministe ", qui enferme des populations dans leurs facteurs de risques - HARSAH, usagers de drogues injectables, etc. - sans tenir compte de leurs capacités propres et donc sans leur offrir d'issue possible. Or la prise en compte des histoires de souffrance déplace, parfois légèrement mais effectivement, les concepts de vulnérabilité et de santé en leur donnant une dimension plus subjective. L'espace des soins devient alors un espace où, malgré les pulsions mortifères, il reste encore possible d'attendre quelque chose de la vie. Cavanaugh introduit le concept d'espace de réapparition des disparus de nos sociétés, espace de résistance face à cette disparition. Cette éthique fondamentale pratique et le concept d'espaces de soins comme espaces de réapparition permettent de considérer un cadre d'intelligibilité original pour les soins pour les personnes vivant avec le VIH/sida, disparues et étrangères.

Le concept d' "étranger par la maladie » semble en effet rendre compte de nombreux mécanismes qui sont présents dans l'infection par le $\mathrm{VIH} /$ sida et isolent les personnes vivant avec le $\mathrm{VIH} /$ sida du reste de la population en les coupant de leurs liens sociaux. Cependant, la réintégration pure et simple de l'étranger dans le groupe des bien-portants n'est pas souhaitable, même si le symptôme qui a provoqué l'étrangeté a disparu ou est devenu socialement acceptable, car on fait alors comme si rien ne s'était passé, comme si l'étrangeté n'avait pas été vécue. Dans certaines circonstances, cette expérience d'étrangeté peut pourtant être une étape structurante dans le processus vital des personnes vivant avec le VIH/sida et, en ce sens, doit être respectée et on doit pouvoir en témoigner. Elle ne doit pas être réduite à néant par des tentatives pour la rendre socialement acceptable.

Les soins peuvent donc être appréhendés comme des espaces de reconstruction, des espaces biographiques où des sujets sont présents avec leur mémoire dangereuse de souffrance, des espaces en construction toujours ouverts aux questions et aux récits.

\section{Les soins comme des espaces de reconstruction et de témoignage}

Prenant en compte les difficultés du témoignage qui conduisent à la disparition de certaines personnes vivant avec le $\mathrm{VIH} /$ sida, nous allons nous pencher maintenant sur les conditions de réapparition et de réintégration de ces étrangers par la maladie dans l'espace social, en nous interrogeant sur les soins et, en particulier, sur la prescription des antirétroviraux. Face à l'épidémie du VIH/sida qui constitue encore un défi majeur sur le plan biomédical, le développement de médicaments antirétroviraux pouvant contrôler efficacement la multiplication du VIH/sida a été l'un des plus rapides de l'histoire de la médecine ${ }^{11}$, transformant en dix ans (1996-2007) une maladie à issue fatale en maladie chronique (Lebouché, Wallach et Routy, 2007).

\section{L'ouverture vers un nouvel espace de vie}

L'usage des thérapies antirétrovirales à ses débuts a eu généralement un impact bénéfique sur l'existence individuelle et sociale des personnes vivant avec le $\mathrm{VIH} /$ sida. Les antirétroviraux remplissent une fonction de normalisation et, par conséquent, ouvrent une possibilité de resocialisation aux individus. De fait, I'amélioration notable de leur état de santé rend possible un retour à la vie normale et au statut social antérieur. Cette resocialisation passe aussi, pour certains, par une reprise des activités sexuelles, souvent après de longues périodes d'abstinence, aussi bien chez ceux qui sont en couple que chez les célibataires (Sow et Desclaux, 2002; Maticka-

\footnotetext{
${ }^{11}$ Les premiers antirétroviraux sont apparus en 1987, soit six ans après l'apparition des premiers cas d'infection par le VIH et trois ans après la découverte de ce virus (Lebouché, Wallach et Routy., 2007).
} 
Tyndale, Adam et Cohen, 2002; Adam, Maticka-Tyndale et Cohen, 2004). Par l'amélioration de l'état de santé qu'ils procurent, les antirétroviraux ont également pour effet de réactiver le désir de procréation chez certaines personnes vivant avec le $\mathrm{VIH} /$ sida, en particulier au sein des communautés africaines et, en France, chez les couples jeunes ou sérodifférents ${ }^{12}$. C'est en ce sens que les antirétroviraux ouvrent de véritables espaces de reconstruction aux personnes vivant avec le $\mathrm{VIH} /$ sida.

\section{De nouveaux espaces dans les pays du Sud}

Les résultats de l'accès aux antirétroviraux sont aussi spectaculaires dans les pays du Sud que dans les sociétés occidentales. Plusieurs études ont évalué l'impact des traitements antisida dans les pays en voie de développement (Ivers et Kendrick, 2005), montrant que dans les pays africains le taux d'adhésion aux antirétroviraux excède habituellement celui de l'Amérique du Nord (Binagwaho et Ratnayake, 2009). Ware et collab. (2009) ont essayé d'expliquer pour quelles raisons on retrouve, contre toute attente, de si bons résultats en Tanzanie, en Ouganda et au Nigeria. Dans l'échantillon des 400 personnes vivant avec le VIH/sida qu'ils ont observées, cette adhésion élevée aux traitements antirétroviraux, renvoie moins à leur volonté d'être en meilleure santé qu'à la protection de leurs relations sociales, en particulier celles entretenues avec l'entourage, ainsi que celle de leur capital social. Les

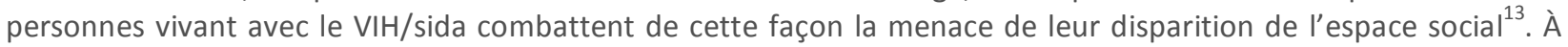
partir des entrevues réalisées auprès d'elles, auprès des treatment partners qui les aident dans la prise des antirétroviraux et auprès des soignants, les chercheurs ont montré que les personnes vivant avec le VIH/sida adhéraient correctement au traitement antirétroviral afin de paraître en bonne santé et faire en sorte que l'entourage ait l'impression que le poids des soins à assurer sera relativement minime. De plus, le fait qu'ils soient en bonne santé encourage l'entourage à leur fournir une aide financière, un soutien pour les déplacements jusqu'à I'hôpital, une collaboration pour l'alimentation adéquate nécessaire afin de limiter les effets secondaires des antirétroviraux, etc. La famille et les amis n'investissent donc pas «à perte " sur quelqu'un qui est proche de la mort ou incurable (Binagwaho et Ratnayake, 2009). De même, les soignants restent disponibles plus longtemps dans le but de maintenir cette amélioration spectaculaire de leur santé. L'ensemble de ces interactions - le capital social - et cette solidarité entre des membres souffrants, leur entourage et leurs soignants, se concentre autour des personnes vivant avec le $\mathrm{VIH} /$ sida, qui sont fortement incitées à prendre leurs traitements et à assumer ainsi leur responsabilité sociale. II est vrai cependant que le contexte africain, où la cohésion sociale est forte, contribue à cette réussite. Les personnes vivant avec le VIH/sida veulent donc vivre, mais aussi garder vivantes leurs interactions sociales et lutter ainsi efficacement contre leur stigmatisation ${ }^{14}$. Toutes ces interactions constituent un véritable corps social intégré autour de la personne vivant avec le $\mathrm{VIH} / \mathrm{sida}$ qui voit ainsi ses liens sociaux maintenus.

Plus globalement, les effets des antirétroviraux dépassent leur efficacité biomédicale. En effet, la disponibilité des traitements dans les pays du Sud contribue à augmenter le recours au dépistage, car un test de VIH positif n'est plus synonyme de mort, mais d'accès aux soins. Farmer et son équipe de Partner in Health ${ }^{15}$, ont ainsi montré comment en Haïti, après un an du programme HIV Inquity Initiative, les demandes de counseling et de dépistage ont augmenté de 300 \% (Mukherjee et Farmer, 2003). Farmer met également en lumière combien la disponibilité des médicaments permet de réintégrer la personne vivant avec le VIH/sida dans la communauté et d'en faire un acteur de prévention essentiel (Arachu et Farmer, 2005). Ces actions ouvrent ainsi aux populations de nouveaux espaces de dépistage, de diagnostic et de soins. Le soin constitue ainsi un espace d’hospitalité où le patient est aidé

\footnotetext{
${ }_{12}^{12}$ Partenaires ayant des statuts sérologiques différents (vis-à-vis du VIH).

${ }^{13}$ En sciences sociales, le capital social se définit comme I'utilisation des relations pour obtenir des bénéfices et réaliser ses projets (Ware et collab., 2009).

${ }^{14}$ Binagwaho et Ratnayake (2009) ajoutent qu’en Amérique du Nord, au contraire, faire adhérer au traitement a toujours été interprétée non comme une responsabilité sociale, mais comme la production d'information et d'explications afin que les patients, à l'échelle individuelle, comprennent ce qu'il faut faire pour rester en vie. Cela a pour corollaire une absence de soutien, et une fragilisation de l'adhésion avec la dépression vécue par la personne vivant avec le VIH/sida.

${ }^{15}$ Partner in Health est une organisation non gouvernementale fondée par l'anthropologue et médecin Paul Farmer. Elle est présente en particulier en Haïti, dans la lutte contre le $\mathrm{VIH} /$ sida et la tuberculose. Elle vise principalement à rendre disponibles les dernières avancées médicales à ceux qui en ont davantage besoin, dont les populations les plus pauvres. Le site est accessible en ligne à http://www.pih.org/index.html (page consultée le 13 décembre 2009).
} 
par le soignant à reprendre le cours de sa vie normale, interrompue par la maladie. Le fait de considérer le soin comme un espace de reconstruction définit la clinique comme un art de la re-possibilisation, dans la mesure où le soin correspond à l'ensemble des moyens offerts par le soignant à son patient afin que ce dernier puisse reprendre son récit de vie et l'intégrer à l'espace social. Le soignant vise alors à faire en sorte que le patient puisse devenir l'acteur du rétablissement de son identité narrative modifiée par l'expérience de la maladie jusque-là (Benaroyo, 2005, p. 81). Ce type de soins est susceptible de faire réapparaître les sujets dans la " normalité » de la vie quotidienne et effacer l'urgence du récit de survie pour orienter ce dernier vers un témoignage plus serein.

\section{Une nouvelle phase dans le récit et le témoignage}

Néanmoins, l'usage des antirétroviraux ne signe pas la fin de l'exception liée au VIH/sida. Elle en oriente plutôt le récit ou le témoignage selon de nouvelles lignes de force. Les nouveaux outils diagnostiques et thérapeutiques rétablissent la suprématie des constructions biomédicales chez les soignants, aux dépens de l'écoute du récit des patients. Les nouveaux médicaments contribuent à l'indétectabilité du VIH et réduisent du même coup les préoccupations liées au contexte de vie des personnes vivant avec le $\mathrm{VIH} / \mathrm{sida}$, les demandes de ces derniers risquant alors de ne plus être entendues (Lebouché et Lévy, 2009).

Cet indéniable succès biomédical complique de nouveau et paradoxalement l'existence des personnes vivant avec le VIH/sida. Ces dernières doivent d'abord apprendre à vivre quotidiennement avec dans leur corps des substances étrangères dont la présence s'impose et exige des stratégies de pratiques autour de leur observance. Elles se retrouvent de plus dans une situation paradoxale : la normalisation de leur existence d'un point de vue médical contraste avec le rappel quotidien de leur statut de séropositif sous traitement. De ce point de vue, la construction et le récit de la maladie se déplacent, puisqu'avec l'arrivée des thérapies les paramètres du témoignage sont en voie d'être redéfinis sans toujours atténuer les conditions de stigmatisation et de discrimination, malgré le fait que les individus sont bien moins à risque de transmettre le $\mathrm{VIH} /$ sida.

\section{Conclusions}

Cette analyse sur la place du récit et du témoignage dans le contexte du VIH/sida permet de préciser la complexité des enjeux éthiques qui accompagnent la problématique du VIH/sida, le statut des personnes vivant avec le $\mathrm{VIH} /$ sida et les pratiques autour des soins et des antirétroviraux. L'expression du récit et du témoignage se heurte pour les personnes vivant avec le VIH/sida à de nombreux obstacles liés au statut de la maladie et aux formes d'exclusion sociale qui l'accompagnent et qui affectent surtout les minorités sexuelles et ethnoculturelles. Particulièrement touchées par cette maladie, ces dernières subissent également des contraintes liées à la communication interculturelle, qui intervient sur les modalités d'énonciation du récit et du témoignage. Les réflexions de Metz et Cavanaugh permettent d'envisager une éthique qui invente des espaces symboliques pour dire la singularité des expériences, le plus souvent non acceptables socialement, afin de lutter contre des pratiques clandestines. Cette éthique contribuerait à réintégrer les personnes vivant avec le VIH/sida dans l'espace social, d'où l'importance de frontières poreuses favorisant des interfaces de rencontres et leur permettant de témoigner non seulement de leur maladie, mais aussi des soins reçus et acceptés au nom du maintien de leurs liens sociaux et de leur réintégration dans leur espace de vie. Dans ces nouveaux contextes, le statut du témoignage et du récit de vie sera valorisé dans la mesure où cela permettra de recentrer les débats entourant les questions de l'invisibilité, de la disparition et de l'étrangeté des personnes vivant avec le VIH/sida.

\section{Références bibliographiques}

Adam, B., Maticka-Tyndale, E. et Cohen, J. (2004). Vivre avec les multithérapies : I'expérience ontarienne. Dans J. Lévy, J. Pierret et G. Trottier (dir.), Les traitements antirétroviraux : expériences et défis (p.153-194). SainteFoy : Presses de l’Université du Québec.

Arachu, A. et Farmer, P. (2005). Understanding addressing AIDS-related stigma : from anthropological theory to clinical practice in Haiti. American Journal of Public Health, 95(1), 53-59.

Arendt, H. (1972 [1961]). La crise de la culture. Paris : Gallimard [coll. Folio essais].

Arendt, H. (2005 [1981]). La vie de l'esprit. Paris : Presses Universitaires de France [coll. Quadrige]. 
Augé, M. (1984). Ordre biologique, ordre social : la maladie, forme élémentaire de l'évènement. Dans $M$. Augé et C. Herzlich (dir.), Le sens du mal : anthropologie, histoire, sociologie de la maladie (p.35-92). Paris : Éditions des Archives Contemporaines.

Bastide, R. (1996). L'événement. Encyclopædia Universalis, 9, 130.

Benaroyo, L. (2005). Responsabilité éthique au quotidien. La narration au cœur du soin. Éthique et Santé, 2(2), 76-81.

Bensa, A. et Fassin, D. (2002). Les sciences sociales face à l'évènement. Terrain, 38, 5-20.

Binagwaho, A. et Ratnayake, N. (2009). The role of social capital in successful adherence to antiretroviral therapy in Africa. PLoS Medicine, 6(1). doi:10.1371/journal.pmed.1000018

Bond, L., Wheeler, D., Millett, G., LaPollo, A., Carson, L. et Liau, A. (2009). Black men who have sex with men and the association of down-low identity with HIV risk behavior. American Journal of Public Health, 99, S92-S95.

Caillé, A. (dir.) (2007). La quête de reconnaissance. Nouveau phénomène social total. Paris : La Découverte. [Revue du MAUSS permanente, 6 nov. 2008, pour la présentation en ligne, http://www.journaldumauss.net/spip.php?article186]

Callahan, D. et Jennings, B. (2002). Ethics and public health: forging a strong relationship. American Journal of Public Health, 2, 169-76.

Canguilhem, G. (2005). Le normal et le pathologique (10 éd.). Paris : Presses Universitaires de France [coll. Quadrige].

Carasso, F. (2001). Primo Levi le malentendu. [En ligne]. http://ecehg.inrp.fr/ECEHG/enjeux-de-memoire/Shoah-etdeportation/reflexions-generales/levi.pdf/view (page consultée le 31 mai 2011).

Certeau (De), M. (1990 [1980]). L'invention du quotidien [Tome 1 : Arts de faire]. Paris : Gallimard [coll. Folio Essais].

David, R. (2004). L'avancée créatrice dans la transition : I’expérience de l’Exil dans le livre de Jérémie. Science et esprit, 56(1), 103-120.

Debru, C. (2005). La vie à l'épreuve de la santé. Entretien avec Sophie Deltin. La Revue des deux mondes, mai, 109-120.

Denzin, N. (1989). Interpretative interactionism. Newbury Park : Sage Publications.

Fink, M. (2009). How AIDS became a Caribbean crisis. The Atlantic, 22 septembre. [En ligne]. http://www.theatlantic.com/doc/200909u/jamaica-aids (page consultée le 30 septembre 2009).

Ivers, L. et Kendrick, D. (2005). Efficacy of antiretroviral therapy programs in resource-poor settings : a metaanalysis of the published literature. Clinical Infectious Diseases, 41, 217-224.

Jobin, G. (2006). L'éthique théologique dans l'espace public contemporain : élucidation des conditions d'insertion du discours théologique dans la sphère publique des sociétés postséculières. Études théologiques et religieuses, 81(3), 337-351.

Keenan, J. (2005). Developments in bioethics from the perspective of HIV/AIDS. Cambridge Quarterly of Healthcare Ethics, 14, 416-423.

Kleinman, A. (1972). Patients and healers in the context of culture. Berkeley : University of California Press.

Ladrière, J. (1996). Théologie et modernité. Revue théologique de Louvain, 27(2), 171-199.

Langlois, E. (2006). L'épreuve du sida. Pour une sociologie du sujet fragile. Rennes : Presses Universitaires de Rennes.

Laplantine, F. (1993). Anthropologie de la maladie. Étude ethnologique des systèmes de représentations étiologiques et thérapeutiques dans la société occidentale contemporaine. Paris : Payot. 
Laplantine, F., Lévy, J., Martin, J.-B. et Nouss, A. (1998). Récit et connaissance. Lyon : Presses Universitaires de Lyon, [coll. Crea].

Lebouché, B., Wallach, I. et Routy, J.-P. (2007). Les traitements antirétroviraux contre le VIH/SIDA : Enjeux et perspectives. Dans J. Lévy et C. Garnier (dir.), La chaîne des médicaments, perspectives pluridisciplinaires (p. 437-468). Québec : Presses de l'Université du Québec.

Lebouché, B. et Lévy, J. (2009). Nouveaux traitements et indétectabilité du VIH. Un risque dans la relation médecinpatient ? Médecines et maladies infectieuses, 39(10), H8-H11.

Le Blanc, G. (2006). Penser la fragilité. Esprit, mars-avril, 249-263.

Lévy, J., Bourdages, A., Bastien, R., Nonn, E., Otis, J., Pelletier, R. et G. Trottier, (2002). Religion, spiritualité et nouvelles thérapies face au VIH/sida. Dans R. Massé et J. Benoist (dir.), Convocations thérapeutiques du sacré (p. 165-182). Paris : Éditions Karthala.

Lévy, J., Pierret, J. et Trottier, G. (2004). Les traitements antirétroviraux. Expériences et défis. Québec : Presses de I’Université du Québec.

Louis-Courvoisier, M. (2005). La greffe du cœur et l'expérience du philosophe. Revue médicale suisse, 1, 264. [En ligne]. http://revue.medhyg.ch/infos/article.php3?sid=1577 (page consultée le 2 décembre 2008).

Maman, M. (2009). Être africain et malade du sida à Paris en 2009. Paroles de patients, paroles de soignants. [En ligne]. http://www.uraca.org/download/editing/search/Uraca-paroles-de-patients-paroles-de-soignants1209.pdf (page consultée le $1^{\mathrm{er}}$ juin 2011).

Maticka-Tyndale, E., Adam, B. et Cohen, J. (2002). Sexual desire and practice among people living with HIV and using combination therapies. The Canadian Journal of Human Sexuality, 11(1), 33-40.

Ménil, A. (1997). Sain(t)s et saufs, Sida : épidémie de l'interprétation. Paris : Belles Lettres.

Mensah, M. (2003). Ni vues ni connues? Femmes, VIH, Medias. Montréal : Les Éditions du remue-ménage.

Metz, J.-B. (1979). La foi dans I’histoire et dans la société. Paris : Éditions du Cerf [coll. Cogitatio Fidei].

Miangu, S. (2008). Perspective interculturelle : comment l’hôpital peut-il soigner autrement? [Éditorial]. Espace éthique/Assitance Publique-Hôpitaux de Paris, 6(3). [En ligne]. http://www.espaceethique.org/fr/popup_result.php?k_doc_lan_pk=309 (page consultée le $1^{\text {er }}$ juin 2011).

Mukherjee, J., Colas, M., Farmer, P., Léandre, F., Lambert, W., Raymonville, M., Koenig, S., et coll. (2003). Perspectives and practice in antiretroviral treatment. Access to antiretroviral treatment and care. Experience of the HIV equity initiative, Cange, Haiti : case study. Genève : Organisation mondiale de la santé.

Nancy, J.-L. (2000). L'intrus. Paris : Galilée [coll. Lignes fictives].

Noali, L. (2009). Espaces d'apparition et espaces de disparition dans le champ carcéral. Champ pénal/Penal Field. [En ligne]. http://champpenal.revues.org/document7135.html (page consultée le 29 septembre 2009).

Parent, A.-M. (2006). Trauma, témoignage et récit : la déroute du sens. Protée, 34(2-3), 113-125.

Pierret, J. (2006). Vivre avec le VIH. Paris : Presses Universitaires de France.

Porée, J. (1993). La philosophie à l'épreuve du mal. Pour une phénoménologie de la souffrance. Paris : Presses universitaires de France.

Sow, K. et Desclaux, A. (2002). Vivre avec un traitement antirétroviral. Dans A. Desclaux, I. Lanièce, I. Ndoye et B. Taverne (dir.), L'initiative sénégalaise d'accès aux médicaments antirétroviraux. Analyses économiques, sociales, comportementales et médicales (p.169-179). Paris : Agence nationale de recherches sur le sida.

Ware, N., Idoko, J., Kaaya, S., Biraro, I., Wyatt, M., Agbaji, O., Chalamilla, G. et Bangsberg, D. (2009). Explaining adherence success in Sub-Saharan Africa: an ethnographic study. PLoS Medicine, 6(1). doi: 10.1371/journal.pmed.1000011 